\title{
$\beta$-Cryptoxanthin modulates the response to phytosterols in post-menopausal women carrying NPC1L1 L272L and ABCG8 A632 V polymorphisms: an exploratory study
}

\author{
F. Granado-Lorencio • L. de las Heras • C. San Millán • F. J. Garcia-López • \\ I. Blanco-Navarro · B. Pérez-Sacristán · G. Domínguez
}

Received: 16 May 2014/Accepted: 11 August 2014/Published online: 28 August 2014

(C) Springer-Verlag Berlin Heidelberg 2014

\begin{abstract}
Phytosterol (PS) intake may be used for hypercholesterolaemia in some groups although the presence of non-responders is well known. Carotenoids and PS/ cholesterol may compete for the same transporters during absorption. As part of a randomized, double-blind, crossover, multiple-dose supplementation study with $\beta$-cryptoxanthin $(\beta-C x)$ and PS, single and combined, polymorphisms of ABCG8 (A632V) and NCPL1 (L272L) were determined in 19 post-menopausal women. Subjects carrying CC polymorphism for NCP1L1 (L272L) showed a net increase in total cholesterol and LDL after PS intake but, interestingly, displayed a decrease in both lipid fractions after consuming PS plus $\beta$-Cx. For the ABCG8 (A632V) gene, CT/TT carriers consuming PS also displayed an increase in total cholesterol and LDL, but this increment was much lower after the intake of PS plus $\beta$-Cx. Additionally, in CC carriers for ABCG8 (A632V), a greater decrease in total cholesterol and LDL was found after the
\end{abstract}

F. Granado-Lorencio $(\bowtie) \cdot$ I. Blanco-Navarro .

B. Pérez-Sacristán

Unidad de Vitaminas, Hospital Universitario Puerta de Hierro-

Majadahonda, 28222 Madrid, Spain

e-mail: fgranado.hpth@salud.madrid.org; fgranado@idiphim.org

F. Granado-Lorencio - L. de las Heras - I. Blanco-Navarro Servicio de Bioquímica Clínica, Hospital Universitario Puerta de Hierro-Majadahonda, 28222 Madrid, Spain

C. S. Millán · G. Domínguez

Servicio de Oncología Médica, Hospital Universitario Puerta de Hierro-Majadahonda, Madrid, Spain

F. J. Garcia-López

Centro Nacional de Epidemiología, Instituto de Salud Carlos III, Madrid, Spain intake of PS plus $\beta$-Cx compared to that observed after PS alone. Overall, our results suggest that $\beta$-Cx improves the response to PS in individuals carrying specific genetic polymorphisms (i.e. non-responders), opening the possibility to modulate the response to PS by food technology. (ClinicalTrials.gov NCT01074723).

Keywords $\beta$-Cryptoxanthin - Phytosterols - Functional food - Genetic variation · Niemann-Pick C1-like protein . ATP-binding cassette G8

\section{Introduction}

Phytosterol (PS) intake may be used for hypercholesterolaemia in some groups since they reduce serum levels of LDL cholesterol (c-LDL) (Gylling et al. 2014). Different studies show that a daily intake of 1.6-2 g/day can reduce intestinal cholesterol absorption by about $30 \%$ and plasma c-LDL levels by $8-10 \%$ (Marangoni and Poli 2010; Ras et al. 2013). However, due to emerging safety issues and the lack of results from trials testing relevant clinical endpoints, guidelines for PS remain controversial (Weingartner et al. 2014).

There is heterogeneity in cholesterol absorption and gene-diet interactions seem to play a role modulating cholesterol/plant sterols absorption and secretion (Izar et al. 2011). Phytosterol absorption is of less magnitude than cholesterol and is preferably secreted in the intestinal lumen by the ATP-binding cassette G5 and G8 (ABCG5/ G8) transporters. Additionally, PS/stanols can reduce cholesterol absorption at the intestinal lumen through the Niemann-Pick C1-like 1 (NPC1L1) transporter pathway by competitive solubilisation in mixed micelles (Izar et al. 2011). 
Traditionally, it has been considered that carotenoids absorption was a simple diffusion process. However, recent studies have demonstrated the presence of proteins involved in the transport of carotenoids into enterocytes. Intestinal transporters involved in absorption and metabolism of carotenoids include NPC1L1, $\beta$ - $\beta$-carotene $15-15^{\prime}$ monooxygenase I (BCMOI), the receptor class $\mathrm{B}$ type I (SR-BI) and the ABCG5 and G8 (Herron et al. 2006; Borel et al. 2007, Borel 2012; Zhao et al. 2008; Lietz and Hesketh 2009; Von Lintig 2010). In this sense, polymorphisms of both ABCG5/G8 and NPC1L1 have been also associated with different responsiveness to PS intervention (Zhao et al. 2008; Teupser et al. 2010), and thus, carotenoids and PS/cholesterol may compete for the same transporters during absorption. This fact could explain, at least in part, the lowering effect on tocopherols and carotenoids observed upon PS consumption (Bañuls et al. 2010)

Within the context of a nutritional intervention with a doubly modified beverage containing $\beta$-cryptoxanthin $(\beta$ Cx) and PS (ClinicalTrials.gov NCT01074723), we found several volunteers who did not respond to PS supplementation and some of them even showed an increase in serum total cholesterol (TC) and c-LDL. Interestingly, these subjects displayed a lower increase or even a decrease in their serum cholesterol and c-LDL levels when they consumed a beverage containing PS plus $\beta-\mathrm{Cx}$ (GranadoLorencio et al. 2014). Thus, as an exploratory study, we assessed the role of genetic polymorphisms as a potential determinant of the response to PS and $\beta-C x$ supplementation.

\section{Materials and methods}

\section{Intervention study}

The study design was a randomized, double-blind, crossover, multiple-dose supplementation study with $\beta-C x$ and PS, single and combined, in healthy post-menopausal women (ClinicalTrials.gov number NCT01074723). Enrolment criteria included age (45-65 years), BMI $<35 \mathrm{~kg} / \mathrm{m}^{2}$, amenorrhoea over 12 months, no hormone therapy, no anabolic or antiresorptive therapy, no use of cholesterol-lowering drugs (i.e. fibrates and statins) and serum levels of fat-soluble vitamins (A, E, D), $\beta-C x$ and biochemical and haematological profile within accepted reference ranges (except for TC and c-LDL, which were considered as inclusion criteria and a "target" endpoint of the intervention). Additional inclusion criteria, sample size calculations, randomization and compliance and supplements composition have been described elsewhere (Granado-Lorencio et al. 2014). The study protocol was approved by the Research Ethics Committee of the Hospital Universitario Puerta de Hierro-Majadahonda (Madrid, Spain), and all subjects were informed and gave their written consent.

A total of 38, apparently healthy, post-menopausal women were finally included although 2 of them refused to participate just after the trial commenced. To assess the biological effect $\beta-\mathrm{Cx}$ and PS, three low-fat milk and fruitbased beverages were developed (Granado-Lorencio et al. 2014) (1) $\beta$-Cx-enriched milk-based fruit drink, (2) PSenriched milk-based fruit drink and (3) $\beta$-Cx-enriched milkbased fruit drink with added PS. $\beta-C x$ is a major dietary provitamin A carotenoid mostly provided by citrus fruits. Thus, mandarin juice was used as the source of $\beta-C x$. The final products were specifically developed to provide $750 \mu \mathrm{g} \beta-\mathrm{Cx}$ and $1.5 \mathrm{~g}$ of PS/250 mL tetra brick (dose for intervention). The intervention study consisted of the supplementation of the diet with $1 \times 250 \mathrm{~mL}$ milk-based fruit drink/day for 4 weeks with a washout period of 4 weeks in between. Overnight fasting blood samples were collected before and after each supplementation period (day 0 and 28) for the analysis of $\beta-\mathrm{Cx}$ in serum (exposition marker) by a quality-controlled ultra-fast liquid chromatography method (Granado-Lorencio et al. 2010) and to assess the effect of $\beta$ $\mathrm{Cx}$ and PS by monitoring changes in the lipid profile [TC, c-LDL and HDL-cholesterol (c-HDL)].

During the study, a modification to genotype some genes related with PS and carotenoid metabolism (i.e. NPC1L1 and ABC G5/G8) was introduced. This sub-study was not initially planned so that 24 subjects could be informed (during the washout period), but only 20 volunteers accepted to provide additional blood samples for lymphocyte isolation and DNA genotyping.

Lymphocytes before and after the intake of the beverages were isolated by standard procedures (Lymphoprep $^{\mathrm{TM}}$, ATOM, AXIS-SHIELD PoC AS, Norway), pooled and stored in liquid nitrogen. Also, due to the limited material available and because this sub-study was performed within a parallel gene expression study, genetic polymorphisms were assayed from RNA and its conversion to cDNA. This approach resulted to be successful for both polymorphisms in 19 volunteers.

\section{RNA extraction and reverse transcription}

Total RNA was extracted using TRIsure reagent (Bioline), and the amount was measured with a NanoDrop spectrophotometer (NanoDrop, Wilmington, DE). For the synthesis of the first-strand cDNA, $400 \mathrm{ng}$ of RNA was reverse-transcribed, using the Gold RNA PCR Core Kit (Applied Biosystems) according to the manufacturer's instructions. Random hexamers were used as primers for cDNA synthesis. 
Table 1 Baseline serum levels of total, LDL cholesterol and $\beta-C x$ according to NPC1L1 L272L and ABCG8 A632 V polymorphisms

\begin{tabular}{|c|c|c|c|c|c|c|c|c|}
\hline & $\begin{array}{l}\text { Total cholesterol } \\
(\mathrm{mmol} / \mathrm{l})\end{array}$ & $P^{*}$ & $\begin{array}{l}\mathrm{c}-\mathrm{LDL} \\
(\mathrm{mmol} / \mathrm{l})\end{array}$ & $P^{*}$ & $\begin{array}{l}\mathrm{c}-\mathrm{HDL} \\
(\mathrm{mmol} / \mathrm{l})\end{array}$ & $P^{*}$ & $\begin{array}{l}\beta-\mathrm{Cx} \\
(\mu \mathrm{mol} / \mathrm{l})\end{array}$ & $P^{*}$ \\
\hline \multicolumn{9}{|c|}{ Beverage containing plant sterols } \\
\hline \multicolumn{9}{|l|}{ NPC1L1 L272L } \\
\hline $\mathrm{CC}(n=9 ; 47 \%)$ & $5.74(0.25)$ & 0.373 & $3.65(0.24)$ & 0.740 & $1.60(0.05)$ & 0.165 & $0.334(0.065)$ & 0.273 \\
\hline $\mathrm{CG} / \mathrm{GG}(n=10 ; 52 \%)$ & $6.03(0.19)$ & & $3.75(0.20)$ & & $1.81(0.13)$ & & $0.439(0.065)$ & \\
\hline All $(n=19)$ & $5.89(0.13)$ & & $3.70(0.15)$ & & $1.76(0.08)$ & & $0.391(0.047)$ & \\
\hline \multicolumn{9}{|l|}{ ABCG8 A632 V } \\
\hline $\mathrm{CC}(n=14 ; 74 \%)$ & $5.74(0.19)$ & 0.205 & $3.59(0.17)$ & 0.341 & $1.68(0.06)$ & 0.284 & $0.372(0.047)$ & 0.471 \\
\hline CT/TT $(n=5 ; 26 \%)$ & $6.18(0.13)$ & & $3.93(0.22)$ & & $1.86(0.24)$ & & $0.449(0.099)$ & \\
\hline All $(n=19)$ & $5.87(0.13)$ & & $3.67(0.14)$ & & $1.73(0.07)$ & & $0.393(0.043)$ & \\
\hline \multicolumn{9}{|c|}{ Beverage containing plant sterols plus $\beta-C x$} \\
\hline \multicolumn{9}{|l|}{ NPC1L1 L272L } \\
\hline $\mathrm{CC}(n=9 ; 47 \%)$ & $5.12(0.31)$ & 0.710 & $3.88(0.32)$ & 0.820 & $1.50(0.05)$ & 0.026 & $0.297(0.058)$ & 0.251 \\
\hline CG/GG $(n=10 ; 52 \%)$ & $6.03(0.26)$ & & $3.77(0.24)$ & & $1.78(0.09)$ & & $0.402(0.065)$ & \\
\hline All $(n=19)$ & $5.95(0.20)$ & & $3.83(0.19)$ & & $1.66(0.06)$ & & $0.351(0.043)$ & \\
\hline \multicolumn{9}{|l|}{ ABCG8 A632 V } \\
\hline $\mathrm{CC}(n=14 ; 74 \%)$ & $5.69(0.25)$ & 0.520 & $3.88(0.32)$ & 0.610 & $1.60(0.05)$ & 0.130 & $0.311(0.054)$ & 0.240 \\
\hline $\mathrm{CT} / \mathrm{TT}(n=5 ; 26 \%)$ & $6.13(0.23)$ & & $3.77(0.24)$ & & $1.81(0.19)$ & & $0.430(0.052)$ & \\
\hline All $(n=19)$ & $5.92(0.19)$ & & $3.80(0.18)$ & & $1.66(0.06)$ & & $0.340(0.052)$ & \\
\hline
\end{tabular}

Values are mean values \pm SEM of volunteers (19 post-menopausal women) classified according to their NPC1L1 and ABCG6 polymorphism variants. Data represent serum concentrations observed at baseline of each intervention

Significant diference is highlighted in bold

* ANOVA test for differences between polymorphisms

Real-time quantitative PCR and genotyping

Quantitative real-time polymerase chain reaction was performed in a Light Cycler apparatus (Roche Diagnostics, Mannheim, Germany) using the LightCycler-FastStart DNA Master SYBR Green I Kit (Roche Diagnostics). Each reaction was performed in a final volume of $20 \mu \mathrm{L}$ containing $2 \mu \mathrm{L}$ of the cDNA product sample, $0.5 \mu \mathrm{M}$ of each primer and $1 \times$ reaction mix including FastStar DNA polymerase reaction buffer, deoxyribonucleotide triphosphates (dNTPs) and SYBR green. Thermal cycling for all genes was initiated with a denaturation step of $95^{\circ} \mathrm{C}$ for $10 \mathrm{~min}$ and then subjected to 40 cycles of PCR (denaturation at $94{ }^{\circ} \mathrm{C}$ for $10 \mathrm{~s}$, annealing at different temperature for each gene for $5 \mathrm{~s}$ and elongation at $72{ }^{\circ} \mathrm{C}$ for $10 \mathrm{~s}$, during which fluorescence was acquired). Primer pairs for ABCG8 A632V and NCPL1 L272L were designed using Primer Express version 2.0 (Applied Biosystems, Foster City, CA). Primers used for PCR for ABCG8 632V were: forward 5'-TGAAGGGCTGATGAAGATTCAG-3' and reverse $5^{\prime}$-CCTGTACTACGTGTCCTTAA -3'; for NPC1L1 L272L, forward 5'-CTCCTGCCAAGACTGTGCTG and reverse 5'-GCTTGTGGGATTCCGTGTGG. The specificity of each primer set was confirmed by melting curve analyses. The PCR products were sequenced using an ABI PRISM 3100-Avant Genetic Analyzer (PE Applied Biosystems, Foster City, CA).

Statistical analysis

Due to the low number of subjects, polymorphisms were aggregated for statistical analysis (Table 1). Also, since no changes in lipid profile was found after consuming the beverage containing just $\beta-\mathrm{Cx}$ (Granado-Lorencio et al. 2014), only the responses after the intake of beverages containing PS and PS plus $\beta$-Cx were assessed according to genetic variants. Data are presented as mean, standard error (SEM) or standard deviation (SD), and the changes in lipid markers according to the polymorphisms after the intake of each beverage were assessed using one-way ANOVA. The statistical software package SPSS release 12.0.1 (SPSS, Inc., Chicago, IL, USA) was used and significance was set at $p<0.5$.

\section{Results}

Age and BMI (mean and SD) of the volunteers were $55.2 \pm 4.1$ years and $26.2 \pm 4.0\left(\mathrm{~kg} / \mathrm{m}^{2}\right)$, and four women were smokers $(21 \%)$. Baseline serum levels of TC, c-LDL, 


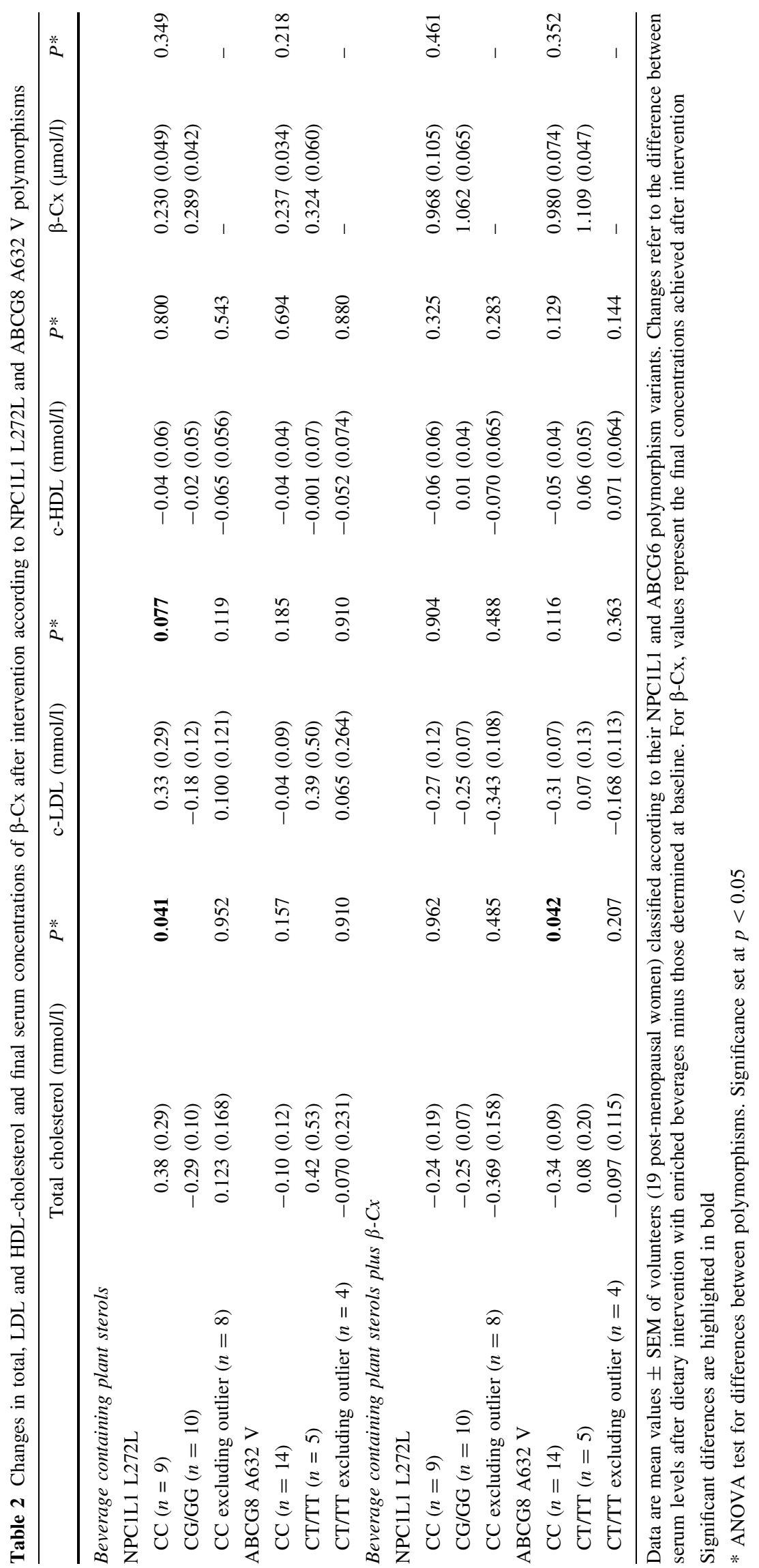


c-HDL and $\beta$-Cx according to genetic polymorphisms are displayed in Table 1. As shown, no statistical differences were found between groups at the start of each intervention, except for c-HDL according to NPC1L1 and before consuming PS plus $\beta-C x$. For each polymorphism, Table 2 shows the mean changes for TC, c-LDL and c-HDL and the final concentrations of $\beta-C x$ achieved in serum after the intake of beverages containing PS and PS plus $\beta-C x$. Compared with baseline values (Table 1), $\beta-\mathrm{Cx}$ levels in serum slightly decreased after PS intake, although the final concentrations did not differ according to the NPC1L1 and ABCG8 variants. On consuming PS plus $\beta-C x$, serum levels of $\beta-\mathrm{Cx}$ increased significantly and to a similar extent for NPC1L1 and ABC G8 polymorphisms.

Considering the change in serum lipids, however, a significant inverse response was observed after the intake of PS depending on the NPC1L1 polymorphism. Thus, CC carriers showed a net increase in TC and c-LDL, but interestingly, these subjects displayed a decrease in both lipid fractions after the intake of the beverage containing PS plus $\beta-C x$, reaching on average a similar reduction as those with $\mathrm{CG} / \mathrm{GG}$ polymorphisms (change not significant according to the genetic variant). For the ABCG8 gene, a similar effect was observed. Subjects consuming PS and carrying CT/TT variants showed an increase in TC and c-LDL compared with CC carriers, although differences did not reach statistical significance. However, after the intake of PS plus $\beta-\mathrm{Cx}$, a much lower increase in serum cholesterol was observed in subjects with CT/TT polymorphisms. Additionally, for $\mathrm{CC}$ carriers, a greater decrease in TC and c-LDL was observed, suggesting that $\beta-C x$ improves the cholesterol-lowering effect of PS in these subjects.

Overall, these findings suggest that $\beta-\mathrm{Cx}$ somehow cancelled out, on average, the effect of $\mathrm{CC}$ polymorphism in NPC1L1 and CT/TT variants in ABCG8 (lack of response) when simultaneously supplied with PS. However, this effect was not observed for all individuals and, as shown in Figs. 1 and 2, the magnitude of these changes may have been skewed by the presence of an outlier (CC for NPC1L1 and CT/TT for ABCG8). Although there were no biological or methodological reasons to exclude this volunteer from the study and her response was unexpected and of great magnitude, the removal of this volunteer from the statistical analysis modified the differences between polymorphisms (Table 2). Thus, for NPC1L1 polymorphisms, differences in lipid fractions decreased upon PS intake, but a greater reduction in serum TC and c-LDL was found after consuming PS plus $\beta-C x$. Similarly, for ABCG8 variants, the exclusion of this subject cancelled the differences between polymorphisms but, consistently, the cholesterol-lowering effect after the intake of PS plus $\beta-\mathrm{Cx}$ improved in CT/TT carriers.
Finally, individual responses to PS and PS plus $\beta-C x$ according to the polymorphisms evaluated are displayed in Figs. 1 and 2. For NPC1L1 (Fig. 1), 13 out of 19 women showed a positive effect [lower increment $(n=4)$, net decrease $(n=3)$ or a higher lowering effect $(n=6)]$ on TC upon intake of PS plus $\beta$-Cx compared to the change observed after PS intake. On the contrary, six subjects showed a similar or lower decrease after the intake of PS plus $\beta$-Cx, while in two participants (GG variant), $\beta-C x$ appears to clearly cancel out the cholesterol-lowering effect of PS. Similarly, for ABCG8, 13/19 show a beneficial effect [lower increment $(n=4)$, net decrease $(n=3)$ or a higher lowering effect $(n=6)]$ on TC after the intake of PS and $\beta$-Cx compared to the change observed after PS consumption. Also, 6 volunteers showed a similar or lower decrease with $\beta-C x$ plus PS and in two subjects (CC, $n=1$; TT, $n=1), \beta-C x$ cancelled out the cholesterollowering effect of PS.

\section{Discussion}

Numerous clinical trials have shown that PS and their esters are effective in reducing circulating cholesterol levels in humans when included in a broad range of food matrices (Ras et al. 2013), although the presence of nonresponders is well known (Izar et al. 2011; Rideaout et al. 2012; Weingartner et al. 2014).

In this study, we found that several volunteers did not respond (non-responders) to PS supplementation and showed even an increase in their cholesterol levels according to polymorphisms in genes involved in intestinal cholesterol absorption and efflux (i.e. ABCG8, NPC1L1). This finding is concordant with studies that have associated genetic variants in these genes and different responses to plant sterols (Izar et al. 2011). For NPC1L1 (L272L), Zhao et al. (2008) observed that mutant $\mathrm{G}$ allele carriers showed a greater reduction in TC and c-LDL compared to wild type counterparts $(\mathrm{C} / \mathrm{C})$, a fact consistent with the decrease in our volunteers after the intake of PS. Similarly, different polymorphisms in the gene for ATP-binding cassette proteins (i.e. A1, B1, G5/G8) have been associated with non-/ irrelevant response and different lowering effect in cholesterol upon PS intake (Zhao et al. 2008; Jakulj et al. 2010). However, while consistent with previous reports, the major finding in the present study is the effect observed upon the intake of the beverage containing PS plus $\beta-C x$, which suggests a potential synergistic effect.

Recent reports indicate that carotenoids can be incorporated via scavenger receptor class B type 1 (SR-BI)mediated pathway, although this may not be the primary mechanism of the incorporation of $\beta-\mathrm{Cx}$ (Takaishi et al. 2012). Other intestinal transporters involved in the 
Fig. 1 Individual changes in serum cholesterol after the intake of beverages containing PS and PS plus $\beta-C x$ according to NPC1L1 (L272L) polymorphism
Fig. 2 Individual changes in serum cholesterol after the intake of beverages containing PS and PS plus $\beta-C x$ according to ABCG8 (A632V)

polymorphism
NPC1L1 L632L
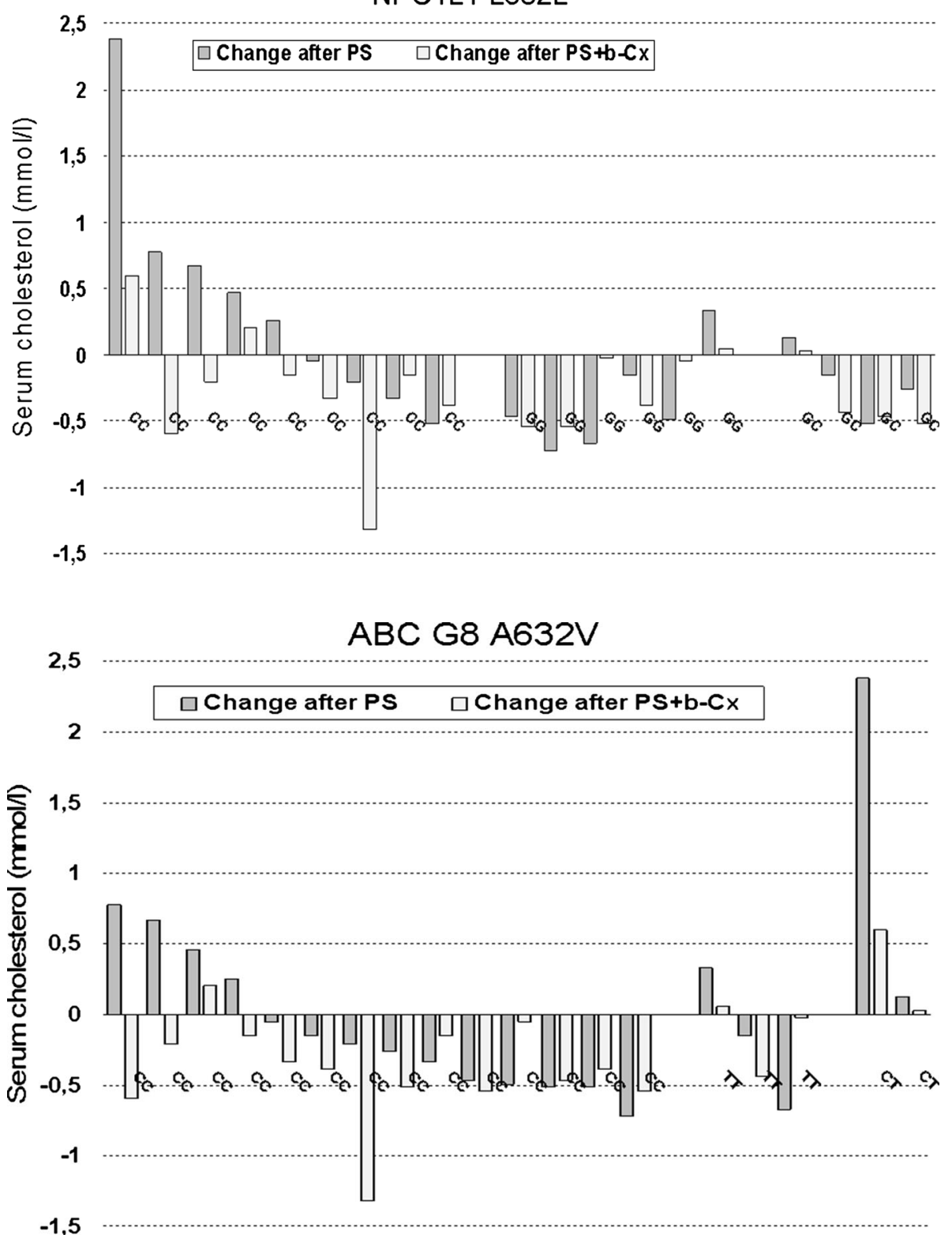

absorption and metabolism of carotenoids include NPC1L1 and the ABCG5/ABCG8 (Herron et al. 2006; Zhao et al. 2008; Lietz and Hesketh 2009). Interestingly, polymorphisms of both ABCG5/G8 and NPC1L1 have been associated with different responsiveness to PS intervention (Zhao et al. 2008; Teupser et al. 2010).

$\beta-\mathrm{Cx}$ can act as ligand for retinoic acid receptor (RAR) and induces genes important for lipid efflux such as the ATP-binding cassette transporters A1 and G1 (ABCA1, ABCG1) (Matsumoto et al. 2007; Fu et al. 2014). Additionally, $\beta-\mathrm{Cx}$ induces in a dose- and time-dependent manner the expression of CYP27A1 (Fu et al. 2014), a fact that may increase 27-OH-cholesterol levels, which in turn may act as an inducer of ABCA1 and ABCG1. Thus, $\beta-C x$ may promote the lipid efflux in macrophages and intestine cells (Li et al. 2007; Fu et al. 2014) and exhibits antiatherogenic effect by inducing CYP27A1 expression via RAR (Fu et al. 2014). It should be noticed, however, that in the present study, serum cholesterol decreased when both PS and $\beta-C x$ were simultaneously supplied and thus, molecular mechanisms involving both intestinal transporters and induction of enzymes of cholesterol catabolism may be involved.

To our knowledge, this is the first study reporting a modulatory effect of $\beta-\mathrm{Cx}$ on the response to plant sterols in humans. Overall, our data suggest that the presence of $\beta$ $\mathrm{Cx}$ improves the response to PS in some individuals carrying specific genetic polymorphisms (i.e. non-responders), 
opening the possibility to counterbalance the response variability by food technology. The randomized crossover design of the study supports our observations and in vitro evidence provides biological plausibility for the observed effects. However, the present findings are based on a low numbers of subjects and the intervention was not specifically designed to assess the effect of genetic polymorphisms so that they should be considered as part of an exploratory study. Additionally, the use of a food-based approach in the present study does not exclude the potential effects of other food components (i.e. flavonoids) despite the lack of biological effect upon the intake of single components (Granado-Lorencio et al. 2014). Thus, the present results should be confirmed in larger, parallel, and longer intervention trials.

Acknowledgments This work was partially funded by AGL200802591-C02-02 (Ministerio de Ciencia e Innovación, Spain). The experimental beverages used in this study were provided by Hero España S.A.

Conflict of interest Granado-Lorencio F, de las Heras L, San Millán, C, Garcia-López, FJ, Blanco-Navarro I, Pérez-Sacristán B and Domínguez $\mathrm{G}$ declare no conflict of interest.

\section{References}

Bañuls C, Martínez-Triguero ML, López-Ruiz A, Morillas C et al (2010) Evaluation of cardiovascular risk and oxidative stress parameters in hypercholesterolemic subjects on a standard healthy diet including low-fat milk enriched with plant sterols. J Nutr Biochem 2:881-886

Borel P (2012) Genetic variations involved in interindividual variability in carotenoid status. Mol Nutr Food Res 56:228-240

Borel P, Moussa M, Reboul E, Lyan B, Defoort C, Vincent-Baudry S et al (2007) Human plasma levels of vitamin E and carotenoids are associated with genetic polymorphisms in genes involved in lipid metabolism. J Nutr 137:2653-2659

Fu H, Wu C, Riaz H, Zhang H et al (2014) Beta-cryptoxanthin uptake in THP-1 macrophages upregulates the CYP27A1 signaling pathway. Mol Nutr Food Res 58:425-436

Granado-Lorencio F, Herrero-Barbudo C, Blanco-Navarro I, PérezSacristán B (2010) Suitability of ultra-performance liquid chromatography for the determination of fat-soluble nutritional status (vitamin A, E, D and individual carotenoids). Anal Bioanal Chem 397:1389-1893

Granado-Lorencio F, Lagarda MJ, Garcia-López FJ, Sánchez-Siles LM, Blanco-Navarro I, Alegría A et al (2014) Effect of b-cryptoxanthin plus phytosterols on cardiovascular risk and bone turnover markers in post-menopausal women: A randomized crossover trial. Nutr Metab Cardiovasc Dis. doi:10.1016/j. numecd.2014.04.013

Gylling H, Plat J, Turley S, Ginsberg HN et al (2014) Plant sterols and plant stanols in the management of dyslipidaemia and prevention of cardiovascular disease. Atherosclerosis 232:346-360

Herron KL, McGrane M, Waters D, Lofgren IE, Clark RM, Ordovas JM, Fernandez ML (2006) The ABCG5 polymorphism contributes to individual responses to dietary cholesterol and carotenoids in eggs. J Nutr 136:1161-1165

Izar MC, Tegani DM, Kasmas SH, Fonseca F (2011) Phytosterols and phytosterolemia: gene-diet interactions. Genes Nutr 6:17-26

Jakulj L, Vissers MN, Tanck MW, Hutten BA, Stellaard H, Kastelein $\mathrm{HH}$ et al (2010) ABCG 5/G 8 polymorphisms and markers of cholesterol metabolism: systematic review and meta-analysis. J Lipid Res 51:3016-3023

Li T, Chen W, Chiang JYL (2007) PXR induces CYP27A1 and regulates cholesterol metabolism in the intestine. J Lipid Res 48:373-384

Lietz G, Hesketh JA (2009) Network approach to micronutrient genetics: interactions with lipid metabolism. Curr Opin Lipidol 20:112-120

Marangoni F, Poli A (2010) Phytosterols and cardiovascular health. Pharmacol Res 61:193-199

Matsumoto A, Mizukami H, Mizuno S, Umegaki K et al (2007) Betacryptoxanthin, a novel natural RAR ligand, induces ATP-binding cassette transporters in macrophages. Biochem Pharmacol 74:256-264

Ras RT, Hiemstra H, Lin Y, Vermeer MA et al (2013) Consumption of plant sterol-enriched foods and effects on plasma plant sterol concentrations-A meta-analysis of randomized controlled studies. Atherosclerosis 230:3336-3346

Rideaout TC, Harding SV, Mackay DS (2012) Metabolic and genetic factors modulating subject specific LDL-C responses to plant sterol therapy. Can J Physiol Pharmacol 90:509-514

Takaishi N, Satsu H, Takayanagi K, Mukai K (2012) In vivo and in vitro studies on the absorption characteristics of $\beta$-cryptoxanthin in the intestine. Biosci Biotechnol Biochem 76:2124-2128

Teupser D, Baber R, Ceglarek U, Scholz M et al (2010) Genetic regulation of serum phytosterol levels and risk of coronary artery disease. Circ Cardiovas Genet 3:331-339

von Lintig J (2010) Colors with functions: elucidating the biochemical and molecular basis of carotenoid metabolism. Annu Rev Nutr 30:35-56

Weingartner O, Baber R, Teupser D (2014) Plant sterols in foods: No consensus in guidelines. Biochem Biophys Res Commun. doi:10. 1016/j.bbrc.2014.01.147

Zhao HL, Houweling AH, Vanstone CA, Jew S, Trautwein E (2008) Genetic variation in ABC G5/G8 and NPC1L1 impact cholesterol response to plant sterols in hypercholesterlolemic Men. Lipids 43:1155-1164 